\title{
Residência Médica em Clínica Médica no Ceará em 2003: Oferta de Vagas e Perfil da Concorrência
}

\author{
Medical Residency in Internal Medicine in \\ Ceará in 2003: Offer of Vacancies and \\ Profile of Candidates
}

Marcelo Gurgel Carlos da Silva ${ }^{1}$ Fernando dos Santos Rocha Filho ${ }^{1}$

PALAVRAS-CHAVE:

- Clínica Médica;

- Internato e Residência;

- Seleção de Pessoal.

KEY-WORDS:

- Internal Medicine;

- Internship and Residency;

- Personnel Selection.

Recebido em: 13/05/2005

Reencaminhado em: 06/11/2006

Reencaminhado em: 31/01/2007

Aprovado em: 13/02/2007

\begin{abstract}
RESUMO
Avaliou-se e comparou-se a oferta de vagas de Residência Médica (RM) em Clínica Médica no Ceará em 2003 e o perfil dos candidatos concorrentes em processos seletivos para estas vagas. Os dados obtidos foram organizados em planilhas eletrônicas. No Ceará, existem 14 instituições, todas em Fortaleza, com programas de RM, das quais apenas três mantêm programa de Clínica. O total de vagas autorizado pela CNRM na área de Clínica Médica é 50, sendo 25 de RI (todas ocupadas). As 314 inscrições para as duas seleções realizadas provinham de 216 médicos (sendo 50,46\% mulheres). A concorrência por vaga na RM de Clínica Médica foi 12,56; para as áreas de especialidades clínicas, a competição por posto foi de 2,54. As instituições universitárias que mais contribuíram foram as federais: do Ceará (44,44\%), da Paraíba (15,28\%) e do Rio Grande do Norte (10,19\%). Por ano de formatura, cerca de 48\% eram recém-graduados de 2002. Conclui-se que é preciso expandir e diversificar a oferta de vagas em residência de Clínica Médica, mediante o esforço combinado das instituições de ensino e das unidades hospitalares; tal medida, no entanto, deveria estar sujeita à apreciação do SUS estadual, ao qual compete definir ou estabelecer parâmetros para nortear a formação médica, incluindo a graduação e a RM, observando perfil e quantidades em função da realidade da saúde cearense.
\end{abstract}

\section{ABSTRACT}

The purpose of this study was to analyze and compare the offer of vacancies for medical residency in Internal Medicine in Ceará, in 2003, along with the profile of the candidates competing for them. The obtained data were organized using electronic spreadsheets. There are 14 institutions offering residency programs in Ceará; most are public and all are headquartered in the State capital, Fortaleza. Only three of them offer medical residency in Internal Medicine. The number of vacancies in Internal Medicine authorized by the CNRM (Regional Medicine Council) is 50, 25 of which are RI (currently filled). Covering three programs in two contests, 216 doctors (50.46\%=women) submitted 314 applications. The competition per vacancy in Internal Medicine was 12.56; for specialized fields it was 2.54. The greater part of candidates were from the medical schools of the federal universities in Ceara (44.44\%), Paraíba (15.28\%) and Rio Grande do Norte (10.19\%). Around 48\% of candidates had graduated recently, in 2002. This study presents a profile of medical residency in Internal Medicine in Ceará. It suggests the need for expanding and diversifying the offer of vacancies through joint efforts of medical schools and hospitals. This, however, should be decided by the Unified Health System (SUS), whose responsibility it is to define or establish parameters for medical education, including graduation and medical residency, adjusting the profile and offer of vacancies to the health situation in Ceará. 


\section{INTRODUÇÃO}

A Residência Médica (RM) é um curso de pós-graduação lato sensu, no qual é dada ao médico a oportunidade de aprofundar conhecimentos e experiências em especialidades específicas. É fundamental por complementar a formação do profissional de Medicina, aprimorando-o para o mercado de trabalho, cada vez mais exigente. Trata-se, pois, de uma formação baseada no ensino em serviço.

Embora a formação médica seja concluída na graduação, a RM aparece como uma forma de aperfeiçoamento profissional, sendo que boa parcela dos médicos recém-formados, seja "por tradição, necessidade de aprimoramento ou até mesmo deficiência da formação profissional", almeja cumprila como fonte de aprendizagem, experiência e especialização, o que vem facilitar a sua inserção no mercado de trabalho ${ }^{1}$.

Na década de 1940, foram iniciados os primeiros programas de RM do Brasil no Hospital dos Servidores do Rio de Janeiro e no Hospital das Clínicas da Universidade de São Paulo $^{2}$, seguindo modelos criados pelos professores Halstedt e Osler, da Universidade Johns Hopkins, no final do século $19^{3}$.

Em 1967, os médicos residentes brasileiros se organizaram e criaram a Associação Nacional dos Médicos Residentes (ANMR), realizando seu I Congresso Nacional4 ${ }^{4}$ Na oportunidade, foram discutidas todas as questões relacionadas aos programas dos cursos, suas qualidades e outras, como a exploração desenfreada do trabalho médico, e os médicos decidiram lutar por uma regulamentação federal.

Em 1977, o governo brasileiro, atendendo às aspirações das universidades e da ANMR, regulamentou a RM brasileira, com o Decreto 80.281, que implantou a Comissão Nacional de Residência Médica (CNRM), constituída de dez membros designados pelo Ministério da Educação e Cultura (MEC). Com este decreto, o presidente Geisel integralizou, no âmbito do MEC, todos os programas de RM do País.

Embora seja subordinada burocraticamente ao Ministério de Educação (MEC), a RM não é parte da universidade, sendo da responsabilidade das instituições e dos serviços de saúde que oferecem o programa1,5.

No estado do Ceará, a primeira experiência de RM ocorreu nos idos de 1962, no então Hospital das Clínicas da Universidade Federal do Ceará (UFC), sob a coordenação dos professores Haroldo Juaçaba e Murilo Martins, que, com outros colegas docentes, abriram programas de Cirurgia Geral e Clínica Médica, respectivamente. Contudo, foi só na segunda metade da década de 1970 que apareceram os primeiros programas no âmbito público estadual cearense, com a iniciativa, em 1976, do antigo Instituto Nacional da Previdência Social (Inamps) em oferecer vagas para áreas cirúrgicas e clínicas ao Hospital Geral de Fortaleza e ao Hospital de Messejana.

Os primeiros programas de RM em Clínica Médica no Ceará surgiram, como referido, na década de 1960, no Hospital das Clínicas da UFC, atualmente denominado Hospital Universitário Walter Cantídio (HUWC). Os médicos-residentes de Clínica Médica, diferentemente dos de Pediatria, iniciavam e concluíam a residência sem ter de sair do Ceará, para completar parte do programa. Na década seguinte, começaram a funcionar os programas de RM em Clínica Médica do Hospital Geral Dr. César Cals (HGCC) e do Hospital Geral de Fortaleza (HGF). A partir de 1978, os programas de RM tutelados pelo estado do Ceará ficaram sob a coordenação da então Fundação de Saúde do Estado do Ceará (Fusec).

Com a criação do Sistema Único de Saúde (SUS), em 1991, houve a fusão dos programas de Residência da Fusec com os do Inamps, e, desde então, toda a atividade relacionada com esta forma de pós-graduação médica, em nosso estado, ficou sob o domínio da Secretaria de Estado da Saúde (Sesa).

Desde 1993, todos os programas de RM do SUS ficaram vinculados funcional e administrativamente à Escola de Saúde Pública do Ceará (ESP-CE), que organiza anualmente o processo seletivo para ingresso à RM, por meio de seu Centro de Coordenação de Residência Médica (Cerme).

A Escola de Saúde Pública do Ceará tem favorecido a feitura de estudos sobre o processo seletivo do SUS, sob a sua alçada6-12; no entanto, a recém-instalada Comissão Estadual de Residência Médica (Cereme), bem como instituições formadoras de médicos e as entidades dessa classe, não contam, até o presente, com um banco de dados consolidado e sistematizado que permita traçar um perfil da oferta e da ocupação de vagas em programas credenciados pela CNRM no estado do Ceará, notadamente tendo em vista as características da demanda que acorre à seleção da RM cearense. Além disso, não pode ser negligenciado que a Cereme deve exercer um papel regulatório no cenário estadual da oferta de vagas, em função das necessidades de saúde do Ceará.

Este trabalho objetiva avaliar e comparar a oferta de vagas de RM em Clínica Médica no Ceará, em 2003, e o perfil dos candidatos concorrentes em processos seletivos para 2003.

\section{MÉTODOS}

Os dados sobre os programas de RM no Ceará foram obtidos mediante acesso eletrônico do site do Ministério da Educação (http://www.mec.gov.br/sesu/CNRM/ consultaCNRM.asp), que abriga a página da Comissão Nacional de Residência Médica (CNRM) e retrata a situação dos programas cadastrados em setembro de 2003. Para cada insti- 
tuição, foram colhidos informes sobre dependência administrativa (federal, estadual, municipal, etc.), município de localização e programas existentes; e de cada programa, a oferta e preenchimento das vagas por ano da RM (R1, R2, R3, etc.), a situação e o ano do término da validade do credenciamento.

Os dados foram dispostos em planilhas eletrônicas Microsoft Excel, software que foi também utilizado para ordenamento e apuração das variáveis de interesse e para a geração das taxas de ocupação, medidas pela relação percentual de vagas preenchidas/vagas credenciadas.

Em obediência à Resolução CNRM 05/2002, os programas de RM foram analisados separadamente: os de acesso direto e aqueles que cobram pré-requisito. Os programas recentemente descredenciados pela CNRM e aqueles em diligência, para fins de credenciamento nessa comissão, foram excluídos da análise dos resultados.

As características dos candidatos foram levantadas diretamente das instituições promotoras dos processos seletivos para RM em 2003, no caso a Escola de Saúde Pública do Ceará (ESP-CE), que conduz o certame para os hospitais estaduais de referência para o SUS, e o Hospital Universitário Walter Cantídio (HUWC), complementados por buscas de informes sobre a escola médica de formação e o ano de formatura junto aos Conselhos Regional de Medicina do Estado do Ceará e Federal de Medicina. Os dados foram igualmente compostos em planilhas eletrônicas Microsoft Excel, que serviram para ordenar, selecionar e classificar os candidatos inscritos nos certames e calcular as taxas de ocupação dos programas e as proporções por variáveis de interesse.

\section{RESULTADOS}

No Ceará, existem 14 instituições, todas sediadas em Fortaleza, com programas de RM, das quais apenas três mantêm programa de Clínica Médica: Hospital Universitário Walter Cantídio (HUWC), Hospital Geral Dr. César Cals (HGCC) e Hospital Geral de Fortaleza (HGF) (Tabela 1).

TABELA 1

Número de vagas e taxa de ocupação da Residência Médica em Clínica Médica no Ceará - 2003

\begin{tabular}{c|c|c|c|c|c|c|c|c|c}
\hline Instituição & \multicolumn{3}{|c|}{ Residentes } & \multicolumn{3}{c}{ Vagas } & \multicolumn{3}{c}{ \%cupação } \\
\hline & $\mathbf{R 1}$ & $\mathbf{R} 2$ & Total & $\mathbf{R} 1$ & $\mathbf{R} 2$ & Total & R1 & R2 & Total \\
\hline HGF & 7 & 7 & 14 & 7 & 7 & 14 & 100,00 & 100,00 & 100,00 \\
\hline HGCC & 6 & 6 & 12 & 6 & 6 & 12 & 100,00 & 100,00 & 100,00 \\
\hline HUWC & 12 & 12 & 24 & 12 & 12 & 24 & 100,00 & 100,00 & 100,00 \\
\hline Total & 25 & 25 & 50 & 25 & 25 & 50 & 100,00 & 100,00 & 100,00 \\
\hline
\end{tabular}

Ao todo, são 54 programas credenciados na CNRM, que oferecem 188 vagas de $\mathrm{R} 1$, das quais 137 correspondem a programas de acesso direto e 51 a programas especializados. O total de vagas autorizado pela CNRM na área de Clínica Médica é 50, sendo 25 de R1 e 25 de R2, e todas as vagas dos programas estão preenchidas, retratando taxa de ocupação de $100,00 \%$ (Tabela 1).
Com referência aos programas especializados, caracterizados pela exigência de cumprimento de programa básico de RM em Clínica Médica, são 29 vagas de R1 em especialidades clínicas, dispostas em Cancerologia Clínica (2), Cardiologia (12), Dermatologia (2), Endocrinologia (2), Gastroenterologia (1), Neurologia (4), Pneumologia (4) e Reumatologia (2) (Tabela 2). 
TABELA 2

Concorrência à Residência Médica em Clínica Médica, segundo programas, no Ceará - 2003

\begin{tabular}{|c|c|c|c|c|}
\hline Programas & Instituição & Candidatos & Vagas & Concorrência \\
\hline Clínica Médica & SUS & 173 & 13 & 13,31 \\
\hline Clínica Médica & HUWC & 141 & 12 & 11,75 \\
\hline Subtotal & & 314 & 25 & 12,56 \\
\hline Cancerologia & HICC & 3 & 2 & 1,50 \\
\hline Cardiologia & $\mathrm{HCM}$ & 17 & 10 & 1,70 \\
\hline Cardiologia & HPC & 3 & 2 & 1,50 \\
\hline Subtotal & & 20 & 12 & 1,67 \\
\hline Dermatologia & HUWC & 15 & 2 & 7,50 \\
\hline Endocrinologia & HUWC & 11 & 2 & 5,50 \\
\hline Gastroenterologia & HGF & 5 & 1 & 5,00 \\
\hline Neurologia & HUWC & 4 & 2 & 2,00 \\
\hline Neurologia & HGF & & 2 & \\
\hline Subtotal & & 4 & 4 & 1,00 \\
\hline Pneumologia & $\mathrm{HCM}$ & 4 & 4 & 1,00 \\
\hline Reumatologia & HGF & 7 & 1 & 7,00 \\
\hline Reumatologia & HUWC & 2 & 1 & 2,00 \\
\hline Subtotal & & 9 & 2 & 4,50 \\
\hline $\begin{array}{l}\text { Subtotal } \\
\text { Especialidades }\end{array}$ & & 71 & 29 & 2,45 \\
\hline Total & & 385 & 54 & 7,12 \\
\hline
\end{tabular}

De 373 candidatos à RM no HUWC, 141 (37,80\%) se inscreveram para Clínica Médica e 32 (8,58\%) para especialidades clínicas, enquanto dos 527 postulantes em RM do SUS, gerenciada pela ESP-CE, 173 (32,45\%) concorriam para Clínica Médica e 33 (6,26\%) disputavam especialidades clínicas. A concorrência por vaga na RM de Clínica Médica foi 12,56, sendo 11,75 no HUWC, e 13,31 no SUS, que seleciona para os programas do HGCC e do HGF; para as áreas de atuação (R3) de especialidades, a competição, por posto, foi de 2,45, com a seguinte especificação, por instituição: Dermatologia $(7,50)$, Endocrinologia $(5,50)$, Neurologia $(2,00)$ e Reumatologia $(2,00)$ no HUWC; Gastroenterologia $(5,00)$ e Reumatologia $(7,00)$ no
HGF; Cardiologia $(1,70)$ e Pneumologia $(1,00)$ no Hospital de Messejana; Cancerologia $(1,50)$ no Hospital do Câncer; e Cardiologia $(1,50)$ no Hospital Prontocárdio (Tabela 2).

As 314 inscrições somadas dos dois processos seletivos, cobrindo os três programas de Clínica Médica, provinham de 216 médicos, já que 98 deles concorreram para Clínica Médi$\mathrm{ca}$, em ambos os processos seletivos. Considerando os diferentes certames de seleção à RM realizados em Fortaleza, 75 (43,35\%), dos 173 inscritos em Clínica Médica no SUS, o fizeram com exclusividade, ao passo que apenas 42 (29,79\%) dentre os 141 registrados em Clínica Médica no HUWC não enfrentaram a outra disputa em Clínica Médica no SUS. Houve 
diferentes arranjos, onde candidatos para Clínica Médica, no SUS, concorreram para Radiologia (4), Pediatria (3), Obstetrícia e Ginecologia (3), Patologia (2), Otorrinolaringologia (2), Cirurgia Geral (1) e Ortopedia e Traumatologia (1) no HUWC, e em Anestesiologia (2) e Cirurgia Geral (1) no Instituto Dr. José Frota (IJF); por outro lado, inscritos para Clínica Médica no HUWC lançaram-se às vagas em Pediatria (2), Medicina de Família e Comunidade (1) e Cirurgia Geral (1) do SUS, e às dos programas de Anestesiologia (6) Cirurgia Geral (5), Orto- pedia e Traumatologia (3) e de Anestesiologia (6) do IJF, e também postularam as de Cirurgia Geral (1), da Santa Casa de Misericórdia (SCM) e Cancerologia Clínica Médica no Hospital do Câncer.

Das 314 inscrições, 162 (51,59\%) eram do sexo feminino, mas, com a exclusão de 98 dos nomes coincidentes em ambos os programas, essa participação cai para 50,46\%; a proporção de candidatas ficou em $53,18 \%$ para o SUS e em $49,65 \%$ para o HUWC (Tabela 3).

TABELA 3

Concorrência à Residência Médica em Clínica Médica, segundo sexo, no Ceará - 2003

\begin{tabular}{l|c|c|c|c|c|c|c|c}
\hline \multirow{2}{*}{ Sexo } & \multicolumn{2}{|c|}{ Combinada } & \multicolumn{2}{c|}{ SUS } & \multicolumn{2}{c}{ HUWC } & \multicolumn{2}{c}{ Total } \\
\cline { 2 - 9 } & No & $\%$ & No & $\%$ & No & No & \% \\
\hline Mas & 107 & 49,54 & 81 & 46,82 & 71 & 50,35 & 152 & 48,41 \\
\hline Fem & 109 & 50,46 & 92 & 53,18 & 70 & 49,65 & 162 & 51,59 \\
\hline Total & 216 & 100,00 & 173 & 100,00 & 141 & 100,00 & 314 & 100,00 \\
\hline
\end{tabular}

Dos 216 médicos interessados em RM de Clínica Médica, as instituições universitárias de formação que mais contribuíram foram a Universidade Federal do Ceará, com 96 (44,44\%), a Universidade Federal da Paraíba, com 33 (15,28\%) e a Universidade Federal do Rio Grande do Norte, com 22 (10,19\%); a Universidade Federal de Alagoas e a Escola de Ciências Médicas de Alagoas participaram com 12 (5,56\%) e 8 (3,70\%) competidores; as universidades federais do Pará e do Piauí entraram com 7 candidatos cada, enquanto outras 14 instituições figuraram com números menores. Segundo macrorregião da unidade formadora do candidato, do Nordeste eram 193 (61,08\%), sendo 97 (44,44\%) do Ceará e 20 de outros estados nordestinos (41,67\%); do Norte foram 17 (5,38\%), do Sudeste $3(0,95 \%)$ e do Centro-Oeste apenas $1(0,32 \%)$. O processo seletivo de Clínica Médica do SUS recebeu candidatos de 13 estados, e o do HUWC, de 14 unidades federativas (Tabela 4). 
TABELA 4

Concorrência à Residência Médica em Clínica Médica, segundo instituição de formação dos candidatos, no Ceará - 2003

\begin{tabular}{|c|c|c|c|c|c|c|c|c|}
\hline \multirow{2}{*}{ Instituição } & \multicolumn{2}{|c|}{ Combinada } & \multicolumn{2}{|c|}{ SUS } & \multicolumn{2}{|c|}{ HUWC } & \multicolumn{2}{|c|}{ Total } \\
\hline & № & $\%$ & № & $\%$ & № & $\%$ & № & $\%$ \\
\hline UFC & 96 & 44,44 & 80 & 46,24 & 75 & 53,19 & 155 & 49,36 \\
\hline UFPB & 33 & 15,28 & 28 & 16,18 & 17 & 12,06 & 45 & 14,33 \\
\hline UFRN & 22 & 10,19 & 17 & 9,83 & 13 & 9,22 & 30 & 9,55 \\
\hline Ufal & 12 & 5,56 & 6 & 3,47 & 8 & 5,67 & 14 & 4,46 \\
\hline ECMAL & 8 & 3,70 & 8 & 4,62 & 1 & 0,71 & 9 & 2,87 \\
\hline UFPA & 7 & 3,24 & 6 & 3,47 & 5 & 3,55 & 11 & 3,50 \\
\hline UFPI & 7 & 3,24 & 6 & 3,47 & 4 & 2,84 & 10 & 3,18 \\
\hline Ufam & 5 & 2,31 & 5 & 2,89 & 3 & 2,13 & 8 & 2,55 \\
\hline UFPE & 5 & 2,31 & 4 & 2,31 & 2 & 1,42 & 6 & 1,91 \\
\hline Uepe & 5 & 2,31 & 5 & 2,89 & - & - & 5 & 1,59 \\
\hline Uepa & 3 & 1,39 & 2 & 1,16 & 2 & 1,42 & 4 & 1,27 \\
\hline UFMA & 2 & 0,93 & 1 & 0,58 & 2 & 1,42 & 3 & 0,96 \\
\hline UFMG & 2 & 0,93 & - & - & 2 & 1,42 & 2 & 0,64 \\
\hline EBMSP & 1 & 0,46 & - & - & 1 & 0,71 & 1 & 0,32 \\
\hline UFBA & 1 & 0,46 & 1 & 0,58 & 1 & 0,71 & 2 & 0,64 \\
\hline UFSE & 1 & 0,46 & 1 & 0,58 & - & 0,00 & 1 & 0,32 \\
\hline UFRR & 1 & 0,46 & 1 & 0,58 & 1 & 0,71 & 2 & 0,64 \\
\hline UDF & 1 & 0,46 & - & - & 1 & 0,71 & 1 & 0,32 \\
\hline Unig & 1 & 0,46 & 1 & 0,58 & 1 & 0,71 & 2 & 0,64 \\
\hline UAM & 1 & 0,46 & 1 & 0,58 & - & - & 1 & 0,32 \\
\hline FMBol & 1 & 0,46 & - & - & 1 & 0,71 & 1 & 0,32 \\
\hline Ign. & 1 & 0,46 & - & - & 1 & 0,71 & 1 & 0,32 \\
\hline Total & 216 & 100,00 & 173 & 100,00 & 141 & 100,00 & 314 & 100,00 \\
\hline
\end{tabular}

Por ano de formatura, 105 (48,61\%) eram recém-graduados de 2002, 43 (19,91\%) foram formados em 2001 e 32 (14,81\%) em 2000; da década de 1990, compareceram 27; um candidato tinha se graduado havia 15 anos, mas 6 concorrentes ainda aguardavam a colação de grau em 2003 (Tabela 5). 
TABELA 5

Concorrência à Residência Médica em Clínica Médica, segundo ano de formatura dos candidatos, no Ceará - 2003

\begin{tabular}{|c|c|c|c|c|c|c|c|c|}
\hline \multirow{2}{*}{ Ano } & \multicolumn{2}{|c|}{ Combinada } & \multicolumn{2}{|c|}{ SUS } & \multicolumn{2}{|c|}{ HUWC } & \multicolumn{2}{|c|}{ Total } \\
\hline & № & $\%$ & № & $\%$ & № & $\%$ & № & $\%$ \\
\hline 1988 & 1 & 0,46 & 1 & 0,58 & 1 & 0,71 & 2 & 0,64 \\
\hline 1990 & 1 & 0,46 & 1 & 0,58 & - & - & 1 & 1,64 \\
\hline 1991 & 1 & 0,46 & - & - & 1 & 0,71 & 1 & 0,32 \\
\hline 1994 & 2 & 0,93 & 2 & 1,16 & 2 & 1,42 & 4 & 1,27 \\
\hline 1995 & 1 & 0,46 & 1 & 0,58 & - & - & 1 & 0,32 \\
\hline 1996 & 3 & 1,39 & 3 & 1,73 & 1 & 0,71 & 4 & 1,27 \\
\hline 1997 & 3 & 1,39 & 3 & 1,73 & 3 & 2,13 & 6 & 1,91 \\
\hline 1998 & 7 & 3,24 & 7 & 4,05 & 2 & 1,42 & 9 & 2,87 \\
\hline 1999 & 9 & 4,17 & 8 & 4,62 & 4 & 2,84 & 12 & 3,82 \\
\hline 2000 & 32 & 14,81 & 31 & 17,92 & 15 & 10,64 & 46 & 14,65 \\
\hline 2001 & 43 & 19,91 & 38 & 21,97 & 31 & 21,99 & 69 & 21,97 \\
\hline 2002 & 105 & 48,61 & 77 & 44,51 & 74 & 52,48 & 151 & 48,09 \\
\hline 2003 & 6 & 2,78 & - & - & 6 & 4,26 & 6 & 1,91 \\
\hline Ign. & 2 & 0,93 & 1 & 0,58 & 1 & 0,71 & 2 & 0,64 \\
\hline Total & 216 & 100,00 & 173 & 100,00 & 141 & 100,00 & 314 & 100,00 \\
\hline
\end{tabular}

\section{DISCUSSÃO}

O Conselho Federal de Medicina e a Associação Médica Brasileira estimulam a procura pelos médicos da pós-graduação lato sensu, o que compreende a RM e os cursos de especialização, com o objetivo de atender à demanda do SUS. O título de especialista é obtido mediante a realização de provas e comprovação de RM.

Com a Resolução CNRM 05/200213, em parte confirmada pela Resolução CFM 1.666/200314, o programa de RM em Clínica Médica teve mantida sua duração de dois anos, e se instituíram outros programas de especialidades clínicas, como Cardiologia, Pneumologia, Neurologia, etc., com dois anos de duração e exigência da feitura prévia de RM em Clínica Médica aos que pretendam cumprir tais programas (http:/ / www.mec.gov.br/sesu/CNRM).

De acordo com a Resolução CNRM 05/200213, os programas de RM credenciáveis pela CNRM poderão ser de acesso direto e com pré-requisito (http:/ /www.mec.gov.br/sesu/ CNRM). O Ceará não dispõe de várias especialidades credenciadas de acesso direto e de outras dependentes de pré-requisitos, havendo tal carência também nas especialidades clínicas. Com efeito, das diversas especialidades clínicas e áreas de atuação da Clínica Médica estabelecidas na Resolução CFM 1.666/200314, existem programas apenas para Cancerologia Clínica, Cardiologia, Dermatologia, Endocrinologia, Gastroenterologia, Neurologia, Pneumologia e Reumatologia; não se conta, por outro lado, com os programas de Alergia e Imunologia, Geriatria, Hematologia e Hemoterapia, Medicina Intensiva, Nefrologia e Nutrologia, que têm o pré-requisito de Clínica Médica. O programa de RM em Hematologia já funcionou no Instituto de Hematologia e Hemoterapia do Ceará (Hemoce), mas foi interrompido.

A inexistência dos programas supramencionados, dirigidos às áreas de atuação da Clínica Médica, não se justifica, em 
sua maioria, perante o estado da arte médica no campo da Clínica Médica no Ceará, considerando a disponibilidade tecnológica das diversas instituições de saúde e dos valores humanos, inteira e facilmente mobilizáveis, para superar, a contento, tais carências. $\mathrm{O}$ esforço coletivo dessas entidades e o de cada instituição, tendo em mente suas especificidades e vocações, concorrerão para mitigar ou sanar tais óbices.

Para ampliar a quantidade de instituições mantenedoras de programas de Clínica Médica, deve-se buscar o concurso de novos parceiros, como o Hospital Geral Dr. Waldemar de Alcântara (HWA) e o Hospital Regional da Unimed (HRU).

Nesse tocante, grosso modo, a título de exemplificação, alguns dos programas em falta, entre aqueles que exigem o pré-requisito de Clínica Médica, poderiam ser assumidos pelas seguintes instituições: Alergia e Imunologia (HUWC); Geriatria (Hospital Regional da Unimed); Hematologia e Hemoterapia (HUWC, em parceria com o Hemoce); Medicina Intensiva (Hospital Regional da Unimed, HGF); Nefrologia (HUWC, HGF), Nutrologia (HUWC).

Os resultados aqui reportados indicam uma concentração absoluta dos programas de Residência Médica na capital cearense. Tal situação traz à pauta de discussão a preocupação com a implantação de centros formadores de médicos nas duas macrorregiões de saúde de Sobral e do Cariri, cujas instituições patrocinadoras (UFC e Sociedade de Ensino Superior do Ceará) argumentavam que concorreria para interiorizar a Medicina no Ceará. Como parte desse processo, considerando o perfil dos acadêmicos desses cursos - que, majoritariamente, não residiam nos municípios-sede antes da admissão no vestibular -, para salvaguardar a promessa de reter os profissionais nessas áreas, é premente oferecer programas de RM nos locais que abrigam os novos cursos do interior cearense. Isto porque, certamente, a RM tem maior potencial de fixar o médico onde ele cumpre o programa, por conta da tendência de a inserção profissional ocorrer mais comumente na vigência desse treinamento ou logo que termine. Para as sedes dessas macrorregiões, à guisa de sugestão, os programas prioritários poderiam ser os de Clínica Médica, Cirurgia Geral, Obstetrícia e Ginecologia, Pediatria e Medicina de Família e Comunidade, sobretudo porque representam programas básicos, de maior amplitude, que vão ao encontro das necessidades de saúde da população, de acordo com a Secretaria da Saúde do Ceará.

Vale apontar que a FMJ conseguiu aprovar junto à CNRM propostas de credenciamento de programas de Medicina de Família e Comunidade, Cirurgia Geral e de Pediatria, com duas vagas cada, e desprezando a oferta em Clínica Médica, que é sobremodo descoberta no interior do Ceará; o mesmo fez a Santa Casa de Misericórdia de Sobral, com a aprovação de programas de Cirurgia Geral e de Clínica Médica, cada um com uma vaga, quantidade tíbia diante do porte desse hospital. Outra medida importante seria a contratação preferencial, por parte das instituições de saúde particulares do interior cearense, dos portadores de certificados de RM realizada nas próprias regiões, como forma de valorizar estes programas de residência e fixar nas regiões os profissionais que os concluíssem. No setor público, poderia ser dado maior peso, nos concursos ou processos seletivos, à contratação de especialistas em Clínica Médica do que em especialidades clínicas, pelas mesmas razões assinaladas, o que não ofende os princípios de eqüidade, garantidos pela Carta Magna do País.

Existe uma perceptível distorção na quantidade de vagas oferecidas nas especialidades clínicas (29 vagas), diante do suprimento de seus respectivos pré-requisitos de Clínica Médica ( 25 vagas), em franco contraste com a baixa taxa de ocupação em algumas especialidades, como Cardiologia e Pneumologia. Isso impõe um redirecionamento de recursos, com o propósito de elevar a oferta de postos em Clínica Médica e adequar a relação Clínica Médica/especialidades clínicas, possivelmente com a canalização das bolsas e da capacidade instalada em favor da formação de maior número de clínicos gerais, a transcorrer no HWA e HRU, preferentemente.

A concorrência de 12,56 candidatos por vaga de RM entre os inscritos para Clínica Médica revela uma condição sobejamente desconfortável, se comparada com a prevalecente em outros programas de acesso direto, a exemplo da vista em Medicina de Família e Comunidade, Infectologia, Psiquiatria, etc., que ostentam pequena competição. Contudo, é preocupante que, mesmo diante de 216 postulantes às 25 vagas - ou seja, sobram mais de sete para cada vaga preenchida -, os programas de especialidades clínicas, em conjunto, têm uma concorrência média de 2,44 inscritos por vaga, valor que seria retraído se fossem expurgadas as múltiplas disputas dos candidatos. Não se trata apenas de excludência quantitativa na Clínica Médica, mas de um forte corte que fulmina candidatos de notável desempenho, passíveis de aprovação caso competissem para outras especialidades de acesso direto, a exemplo de Medicina de Família e Comunidade, Infectologia, Psiquiatria, etc., entre outras $7,8,10$.

Os valores aqui retratados possivelmente refletem uma situação bastante desfavorável, pois a concorrência dos aqui graduados pela UFC representa menos da metade, ficando cerca de $55 \%$ na conta dos egressos de outras escolas médicas; como é cada vez menor o contingente dos formados na UFC que logra êxito em processos seletivos de programas de RM de outros estados brasileiros, como Rio de Janeiro e São Pau- 
lo, ao passo que é crescente a busca por vagas na RM do Ceará, da parte dos formados em outros estados brasileiros, a situação tende ao acirramento da competição, notadamente com a chegada dos médicos diplomados pelas novas escolas médicas do Ceará a partir de 2006.

É vital que, além das entidades patrocinadoras ou parceiras das recém-criadas escolas médicas, outras unidades hospitalares elaborem propostas e se qualifiquem para montar programas de RM. No último caso, vale alertar que o Hospital Geral Dr. Waldemar de Alcântara reúne pré-condições, aliás bastante favoráveis, para submeter à CNRM projetos de instalação de RM em Clínica Médica, minorando um dos pontos de estrangulamento da formação especializada; também como exemplo, a inserção do Hospital Regional da Unimed (HRU) nessa modalidade de pós-graduação, considerando sua missão e vocação diferenciada em vários serviços especializados, poderia aportar distintos programas.

É conveniente assinalar que, considerando que os prazos para encaminhamento de propostas e apreciação na CNRM exigem longa antecipação, é urgente aglutinar esforços coletivos com vistas a corrigir o desequilíbrio que cronologicamente não está tão longe, antes que seus efeitos nefastos se tornem realidade.

Vale salientar que a implantação de programas de RM significa um investimento na qualidade dos serviços prestados que gera benefícios aos usuários e aos provedores desses serviços; desse modo, qualquer instituição, pública ou privada, que condicione ou privilegie a absorção de pessoal com essa titulação, sob as diferentes formas de inserção funcional, incluindo as de terceirização, deve, como contrapartida, incentivar ou patrocinar a instalação de programas de RM.

Sublinhe-se que a definição da oferta de vagas de RM deveria ser regulada pelos gestores do SUS, tendo por base a realidade de saúde do País e suas especificidades regionais, como sucede em alguns países desenvolvidos (Inglaterra e Holanda), ao invés de se submeter quase exclusivamente aos ditames de mercado, como ora ocorre de forma predominante.

Os vistosos números de inscritos em certas áreas de RM retratam a interferência de mecanismos de mercado na motivação dos candidatos em suas respectivas escolhas. Isto está em clara dissonância com as demandas em saúde da população e, por vezes, em discordância com as diretrizes educacionais da formação médica e até com o próprio projeto políticopedagógico de seu curso de formação. Como se sabe, os novos cursos médicos instalados no Ceará foram elaborados tendo por norte a formação de médicos para o SUS, com ênfase na estratégia da Saúde da Família. No entanto, nas primeiras turmas de graduados, tem-se notado que esses egressos têm o interesse mais voltado para a atividade privada.
Em 1997, em Brasília, realizou-se o I Seminário de Residências Médicas, em que se discutiu a importância de pautar a formação da RM em função das necessidades do SUS. Ficou evidenciado o círculo vicioso que envolve a universidade que não muda o profissional porque o SUS - que também requer um profissional com outro perfil - também não se modifica. A corporação médica presente ao evento concluiu que o SUS não interferia na definição de vagas de $\mathrm{RM}^{15}$.

O estado do Ceará divulgou, em 26/10/2005, o edital do concurso público unificado para preenchimento de 4.319 vagas destinadas a médicos, enfermeiros e cirurgiões-dentistas do Programa Saúde da Família (PSF) em 118 municípios cearenses. A decisão veio ao encontro da reclamação das entidades dessas categorias profissionais, notadamente as dos médicos, que, de longa data, denunciavam a precarização da forma contratual de trabalho, dominante nas equipes do PSF. Os atrativos salariais e a nomeação efetiva foram fundamentos importantes para a regularização do trabalho médico nas equipes de Saúde da Família e para a implementação do PSF cearense.

No mesmo período, no intuito de redirecionar a demanda profissional para o PSF, a Secretaria de Saúde de Fortaleza (SSF) apresentou à CNRM proposta de credenciamento de programa de RM em Medicina de Família e Comunidade, com 200 vagas anuais, logrando autorização para o funcionamento inicial, em 2006, com 75 vagas. Para despertar maior interesse da clientela, a SSF duplicou o valor monetário da bolsa paga ao residente e instituiu pagamento aos coordenadores, supervisores e preceptores do programa. Espera-se que, com essas ações, se possa dispor de um profissional bem formado e mais bem preparado para a inserção no PSF.

\section{CONCLUSÃO}

Este trabalho permitiu traçar o perfil da RM no Ceará, enfocando aspectos da oferta e ocupação de vagas, e dos programas credenciados na área da Clínica Médica junto à CNRM, analisando diversas características desses programas, como local de realização, especialidade e tipo de acesso.

No Ceará, existem 14 instituições, todas sediadas em Fortaleza, com programas de RM, das quais apenas três mantêm programa de Clínica Médica: Hospital Universitário Walter Cantídio (HUWC), Hospital Geral Dr. César Cals (HGCC) e Hospital Geral de Fortaleza (HGF). O total de vagas autorizado pela CNRM na área de Clínica Médica é 50, sendo 25 de R1, e todas estão ocupadas.

A concorrência por vaga na RM de Clínica Médica foi 12,56, sendo 13,31 no HUWC e 11,75 no SUS (HGCC e HGF); para as áreas de especialidades clínicas, a competição por posto foi de 2,54 . 
As 314 inscrições somadas dos dois processos seletivos, cobrindo os três programas de Clínica Médica, provinham de 216 médicos; delas, 161 (51,59\%) eram do sexo feminino, mas, com a exclusão de 98 dos nomes coincidentes em ambos os programas, essa participação cai para $50,46 \%$; a proporção de candidatas ficou em $53,18 \%$ para o SUS e em $49,65 \%$ para o HUWC.

Dos 216 médicos interessados em RM de Clínica Médica, as instituições universitárias de formação que mais contribuíram foram a Universidade Federal do Ceará (44,44\%), a Universidade Federal da Paraíba $(15,28 \%)$ e a Universidade Federal do Rio Grande do Norte (10,19\%). Por ano de formatura, cerca de 48\% eram recém-graduados de 2002.

Esses valores assinalam a franca necessidade de expandir e diversificar a oferta de vagas de RM na área da Clínica Médica no Ceará, mediante o esforço combinado das instituições de ensino e das unidades hospitalares, incluindo as detentoras de programas credenciados e novas entidades dotadas de qualificação para cumprir essa missão, de modo não apenas a superar dificuldades da educação pós-graduada em Medicina, mas, sobretudo, propiciar melhor habilitação aos médicos recém-graduados e, desse modo, garantir maior resolutividade ao SUS do Brasil.

Enfim, é imperativo esclarecer que os aspectos pontuais aqui expostos deveriam estar sujeitos à apreciação das instâncias superiores do SUS estadual, ao qual compete definir ou estabelecer parâmetros para nortear a formação médica, incluindo a graduação e a RM, observando perfil e quantidades, em função da realidade da saúde cearense, identificada mediante estudos específicos, corroborados por seus indicadores de saúde.

\section{REFERÊNCIAS}

1. Machado MH. Os médicos no Brasil: um retrato da realidade. Rio de Janeiro: Fiocruz; 1997.

2. Sousa EG. A residência médica no Brasil. Rev Bras Educ Med. 1985; 9(2): 112-4.

3. Furtado T. Residência médica e mestrado na área profissional da medicina. Rev Bras Educ Med. 1995; 9(1): 5-6.

4. Leite EV. Residência médica, mestrado e doutorado. Rev Bras Educ Med. 1983; 7(2): 101-4.

5. Feuerrwerker LCM. Mudanças na educação médica e residência médica. São Paulo: Hucitec/Rede Unida; 1998.

6. Machado MH, coord. Perfil dos médicos no Brasil: relatório final. Rio de Janeiro: NERHUS; 1996.

7. Silva JDM, Silva LFG, Silva MGC. Características educacionais dos candidatos ao processo seletivo da residência médica do sistema único de saúde do estado do Ceará para ingresso em 1997. Rev Ceara Med. 2000; 9(1): 1-8.

8. Silva MGC, Silva JDM, Silva LFG. Avaliação dos critérios de seleção dos candidatos ao processo à residência médica do sistema único de saúde do estado do Ceará para ingresso em 1997. Rev Ceara Med. 2000; 9(3): 1-9.

9. Silva JDM, Silva LFG, Silva MGC. Análise curricular e desempenho dos candidatos ao processo seletivo da residência médica do sistema único de saúde do estado do Ceará para ingresso em 1997. Rev Med Univ Fed Ceara 2001; 41(1-2): 61-77.

10. Silva JDM, Silva LFG, Silva MGC. Aspectos sócioeconômicos dos candidatos à residência médica do sistema único de saúde do estado do Ceará para ingresso em 1997. Rev Bras Educ Med. 2002; 26(1): 13-20.

11. Mariano REM, Silva MGC. O processo seletivo para a residência médica do sistema único de saúde no Estado do Ceará. In: Jorge MSB, Sampaio HAC, orgs. Construção do conhecimento em saúde coletiva: políticas públicas e diversidades. Fortaleza: Ed. INESP/EdUECE;2001. p. 249-60.

12. Mariano REM, Silva MGC. O processo de avaliação na formação do profissional de saúde. In: Silva MGC, Jorge MSB, orgs. Construção do saber em saúde coletiva: transição epidemiológica e diversidades. Fortaleza: Ed. INESP/ EdUECE; 2002. p. 348-58.

13. Brasil. Ministério da Educação. Comissão Nacional de Residência Médica .Resolução CNRM 05/2002. [online]. [capturado 15 set. 2003]. Disponível em: http:// www.mec.gov.br/sesu/CNRM/consultaCNRM.asp.

14. Conselho Federal de Medicina. Resolução CFM 1.666/2003. [online] Brasília, DF. [capturado 15 set. 2003]. Disponível em: http://www.mec.gov.br/sesu/CNRM/ consultaCNRM.asp.

15. Ros MA, Pierantoni CR, Haddad AR, Ribeiro C, Severo DO, Souza TT. Residência multiprofissional em saúde da família: uma conquista do movimento sanitário. Cad $\mathrm{RH}$ Saude 2006; 3(1): 109-18.

\section{Conflito de Interesse}

Declarou não haver.

\section{Endereço para correspondência}

Fernando dos Santos Rocha Filho

Rua Deputado João Pontes - 851 - apto 901

60040-430 Fortaleza - CE.

e-mail: fernando.dos.filho@terra.com.br 